\title{
PIK3R3 Promotes Metastasis of Pancreatic Cancer via ZEB1 Induced Epithelial- Mesenchymal Transition
}

\author{
Yun-Peng Peng ${ }^{a, b} \quad$ Yi Zhu ${ }^{a, b} \quad$ Ling-Di Yin ${ }^{a, b} \quad J i-S h u$ Wei ${ }^{a, b} \quad$ Xin-Chun Liu \\ Xiao-Le Zhu ${ }^{a, b}$ Yi Miao a,b \\ aPancreas Centre, First Affiliated Hospital, Nanjing Medical University, Nanjing, bPancreas Institute, \\ Nanjing Medical University, Nanjing, People's Republic of China
}

\section{Key Words}

Pik3r3 • Pancreatic cancer • Epithelial-mesenchymal transition • ZEB1 • Metastasis

\begin{abstract}
Background/Aims: PIK3R3 is a regulatory subunit of phosphatidylinositol 3-kinase (PI3K) which plays an essential role in the metastasis of several types of cancer. However, whether PIK3R3 can promote the metastasis of pancreatic cancer (PC) is still unclear. In this study, we characterized the role of PIK3R3 in metastasis of PC and underlying potential mechanisms. Methods: RT-PCR, western blot, immunofluorescence (IF) and immunohistochemistry (IHC) were applied to investigate the expression of genes and proteins in different cell lines and tissues. To assess the function of PIK3R3 and related mechanisms, the cells with RNAimediated knockdown or overexpression were used to perform a series of in vitro and in vivo assays. Results: PIK3R3 was significantly overexpressed in pancreatic cancer tissues, especially in metastatic cancer tissues, as well as in pancreatic cancer cells. Functional assays suggested that overexpression or knockdown of PIK3R3 could respectively promote or suppress the migration and invasion of PC cells in vitro and in vivo. Further mechanism related studies demonstrated that ERK1/2-ZEB1 pathway-triggered epithelial-mesenchymal transition (EMT) might be responsible for the PIK3R3-induced PC cell migration and invasion. Conclusion: PIK3R3 could promote the metastasis of PC by facilitating ZEB1 induced EMT, and could act as a potential therapeutic target to limit PC metastasis.

\section{Introduction}

Pancreatic cancer (PC) is the fifth leading cause of cancer-related death worldwide, with a 5-year relative survival of only $8 \%$ and a median survival of 6 months [1]. The morbidity of this cancer is persistently increased over the past decades; however, the survival rate is not significantly improved [2]. The only available therapy for PC is radical resection which

Y.-P. Peng, Y. Zhu and L.-D. Yin contributed equally to this work. 
prolongs survival and offers a potential cure [3]. However, the surgical resection rate is less than $20 \%$ due to the frequent occurring of local advance and distant metastasis [4]. Therefore, investigating the potential mechanisms of PC invasion and metastasis may contribute to the exploration of novel therapeutic strategy $[5,6]$.

Epithelial-mesenchymal transition (EMT) and MMPs were widely reported to be responsible for the invasion and metastasis of PC cells [7, 8]. EMT is an essential phenotypic conversion from epithelial to mesenchymal phenotype during embryonic development, and is significantly correlated with cancer invasion and metastasis [9]. The conversion of EMT is promoted by transcription factors (such as SNAIL, SLUG, TWIST1, ZEB1, and ZEB2) which can be triggered by several endogenous and exogenous factors via specific signaling pathways [10-12].

PIK3R3 is a regulatory subunit of phosphatidylinositol 3-kinase (PI3K) [13]. Several studies have reported that PIK3R3 is abnormally overexpressed in some types of cancer, such as lung cancer, colorectal cancer, and gastric cancer [14-16]. Data obtained from clinical samples suggested that overexpression of this molecule is significantly associated with the progression and prognosis of certain cancers, and functional assays demonstrated that PIK3R3 could promote the proliferation, migration, and invasion of some cancers in vitro and vivo [17-19]. Some studies have mentioned the role of PIK3R3 in PC [20, 21]. However, the function and mechanism of PIK3R3 in PC is still unclear, especially the function of PIK3R3 in tumor metastasis. Therefore, in the present study we assessed PIK3R3 expression in PC tissues and cell lines, and further investigated the role and mechanisms of PIK3R3 in PC cell metastasis.

\section{Materials and Methods}

\section{Patients}

25 pairs of primary cancer and adjacent tissues were obtained from patients diagnosed with PC at Pancreas Center, The First Affiliated Hospital of Nanjing Medical University from 2013 to 2015, as well as 25 metastatic cancer tissues. 25 metastatic cancer tissues were obtained from metastatic sites (patients without radical operation), and primary cancer tissues were obtained from the primary sites (patients with radical operation). Primary cancer and adjacent tissues were obtained from radical resection specimens, and metastatic cancer tissues were got from biopsy specimens of distant metastases. All tissues applied in this study were fixed in formalin, embedded paraffin, and further used to IHC. Our study was approved by the Ethics Committee of the First Affiliated Hospital of Nanjing Medical University.

\section{Immunohistochemistry (IHC) Staining and Assessment}

IHC was performed according to a standard immunoperoxidase staining procedure [22] . Two experienced pathologists who were blinded to enrolled patients viewed the tissue sections independently. The methods for IHC assessment applied in our previous study were used here [23]. All antibodies used here were all purchased from Abcam (Cambridge, MA, USA).

\section{Cells and Cell Culture}

The normal human pancreatic ductal cell line hTERT-HPNE and all pancreatic cancer cell lines were available in our laboratory. hTERT-HPNE cell line was cultured based on the recommendation of ATCC (Rockville, MD, USA). All PC cell lines mentioned above were cultured in DMEM supplemented with $10 \%$ FBS, penicillin $(100 \mathrm{U} / \mathrm{mL})$ and streptomycin $(100 \mu \mathrm{g} / \mathrm{mL})$.

\section{Stable transfection of PC cells}

Recombinant lentivirus for PIK3R3 overexpression and knockdown were purchased from Genepharma, Shanghai, China. Human PIK3R3 coding sequencer was amplified and cloned into a pGLV3/H1/GFP+Puro vector to further generate PIK3R3 overexpression lentivirus. Biologically active short hairpin RNAs (ShRNA) were applied to generate PIK3R3 knockdown lentivirus. The ShRNA target sequence for human PIK3R3 used in this study were shown as follows: 5'-GGGAGGAGGTAAATGACAAAT-3', 5'-GGGAATTAAGAATGAGGATGC-3', 


\section{Cellular Physiology Cell Physiol Biochem 2018;46:1930-1938 \\ \begin{tabular}{l|l} 
and Biochemistry Published on/000489382 2018 & $\begin{array}{l}\text { (c) } 2018 \text { The Author(s). Published by S. Karger AG, Basel } \\
\text { www.karger.com/cpb }\end{array}$ \\
\hline
\end{tabular}}

Peng et al.: PIK3R3 Promotes Metastasis of Pancreatic Cancer via ZEB1

5'-GGTTTGTTGAGGATATCAATC-3'. Mia PaCa-2 and BxPC-3 cells were respectively transfected with PIK3R3 overexpression and knockdown lentivirus. Puromycin was used to select the stably transfected cells. The efficiency of PIK3R3 overexpression and knockdown were detected by qRT-PCR and Western blot.

\section{Quantitative real time reverse transcription polymerase chain reaction}

Trizol reagent was used to extract total RNA from different cells. PrimeScript RT Master Mix was applied to reverse-transcribe total RNA into cDNA. The Step One Plus Real-Time PCR System (Applied Biosystems, Carlsbad, CA, USA) and FastStart Universal SYBR Green Master were used to perform the qRTPCR amplification. All relevant experiments here were conducted based on the instructions provided by manufacturer. Primer Premier 5 and Oligo 6 were respectively applied to design and check the specific primers for human PIK3R3, ZEB1, and $\beta$-ACTIN. The sequences of primers are as followed: PIK3R3 (sense, 5'-CTTGCTCTGTGGTGGCCGAT-3' and antisense, 5'-GACGTTGAGGGAGTCGTTGT-3'), ZEB1 (sense, 5'-GATGATGAATGCGAGTCAGATGC-3' and antisense, 5'-ACAGCAGTGTCTTGTTGTTGT-3'), Beta-Actin (sense, 5'-AGCGAGCATCCCCCAAAGTT-3' and antisense, 5'-GGGCACGAAGGCTCATCATT-3'). The relative expression of certain mRNA was normalized by formula $2^{-\Delta \Delta t}$ (Ct means the cycle threshold).

\section{Western blotting}

Total protein was extracted by using a Total Protein Extraction Kit which containing PMSF, protease inhibitors, and phosphatase inhibitors. Extracted protein was mixed with $5 \times$ SDS, and further degenerated in boiling water for $5 \mathrm{~min}$. Western blot analysis was performed according to the standardized methods. AntiPIK3R3 (ab97862), E-Cadherin (ab133597), Vimentin (ab137321), SNAIL (ab53519), SLUG (ab85936), ZEB1 (ab124512), ZEB2 (ab138222) antibodies were all purchased from Abcam (Cambridge, MA, USA). Anti-ERK1/2 (\#4695) and p-ERK1/2 (\#4370) antibodies were obtained from Cell Signaling Technology (Danfoss, MA, USA).

\section{Immunofluorescent assay}

Cells were seeded on the 12-well Cell Culture Plates and cultured for $24 \mathrm{~h}$. Cells were washed with PBS and fixed in stationary liquid for 15 minutes at room temperature. And then, cells were washed with PBS for three times after discarding stationary liquid. Blocking Buffer for immunocytofluorescence was added to the plate for $1 \mathrm{~h}$ at room temperature. Primary antibodies were added to the plate and incubated at $4{ }^{\circ} \mathrm{C}$ overnight, followed by incubation with $\mathrm{Cy}^{\mathrm{TM}} 3$ labeled secondary antibodies (Jackson Laboratory) for $1 \mathrm{~h}$ at room temperature. Nuclei was further stained with DAPI for $5 \mathrm{~min}$ at room temperature. A fluorescence microscopy was applied to photograph Fluorescence images. Anti- E-Cadherin and Vimentin antibodies were all purchased from Abcam (Cambridge, MA, USA).

\section{Migration and Invasion assays}

In vitro cell invasion and migration ability were respectively evaluated by using Matrigel-coated and non-Matrigel-coated transwell filters (BD Biosciences) as described in previous study [24]. The cells for migration assays were harvested after $24 \mathrm{~h}$, and the cells for invasion were harvested $48 \mathrm{~h}$.

\section{Animal studies}

All female BALB/c nude mice aged 5 weeks used in this study were purchased from Model Animal Research Center of Nanjing University. To assess the prometastatic function of PIK3R3 in vivo, fresh cancer cells $\left(1 \times 10^{6}\right.$ per mice) were implanted into the pancreas under anesthesia which was induced by $1 \%$ pentobarbital sodium. After four weeks of tumor implantation, all nude mice were sacrificed. Primary and metastatic tumors were obtained from the mice to photograph images, and then, fixed in formalin for further paraffin embedding.

\section{Statistical analysis}

Independent experiments were performed three times to generate respective data in each figure. The Mann-Whitney U-test was applied to compare PIK3R3 expression in pancreatic cancer and paired adjacent tissues. The comparison between two groups was done by independent Student's t-test. SPSS 20.0 software were used to perform all the analyses. All data were expressed as mean \pm SD. Differences were considered statistically significant at $\mathrm{P}<0.05$. 


\section{Results}

Aberrantoverexpression of PIK3R3 in PC tissue and cell lines

IHC analyses suggested that the expression of PIK3R3 in primary and metastatic cancer tissues was significantly higher than that in adjacent tissues, while PIK3R3 expression in metastatic tissues was the highest (Fig. 1A). Such results suggested that PIK3R3 might be associated with PC metastasis.

Then, the expression levels of PIK3R3 in seven pancreatic cancer cell lines were evaluated using qRTPCR and Western blot, including AsPC-1, Mia PaCa2, SW1990, BxPc-3, Capan-2, CFPAC-1, and PANC-1 (Fig. 1B). Compared to the normal human pancreatic ductal cell hTERT-HPNE, almost all cancer cell lines expressed increased levels of PIK3R3. And then, Mia PaCa-2 (highest) and BxPc3 (lowest) were respectively selected to establish PIK3R3 knockdown and overexpression cell lines. Stable PIK3R3 knockdown and overexpression cell lines were established and further confirmed via qRTPCR and Western blot (Fig. 1C).

PIK3R3 promotes the migration and invasion of PC cells

Matrigel-coated and n on-Matrigel-coated transwell filters were applied to evaluate the migration and invasion of these cells in vitro. As shown in (Fig. 2A), the numbers of

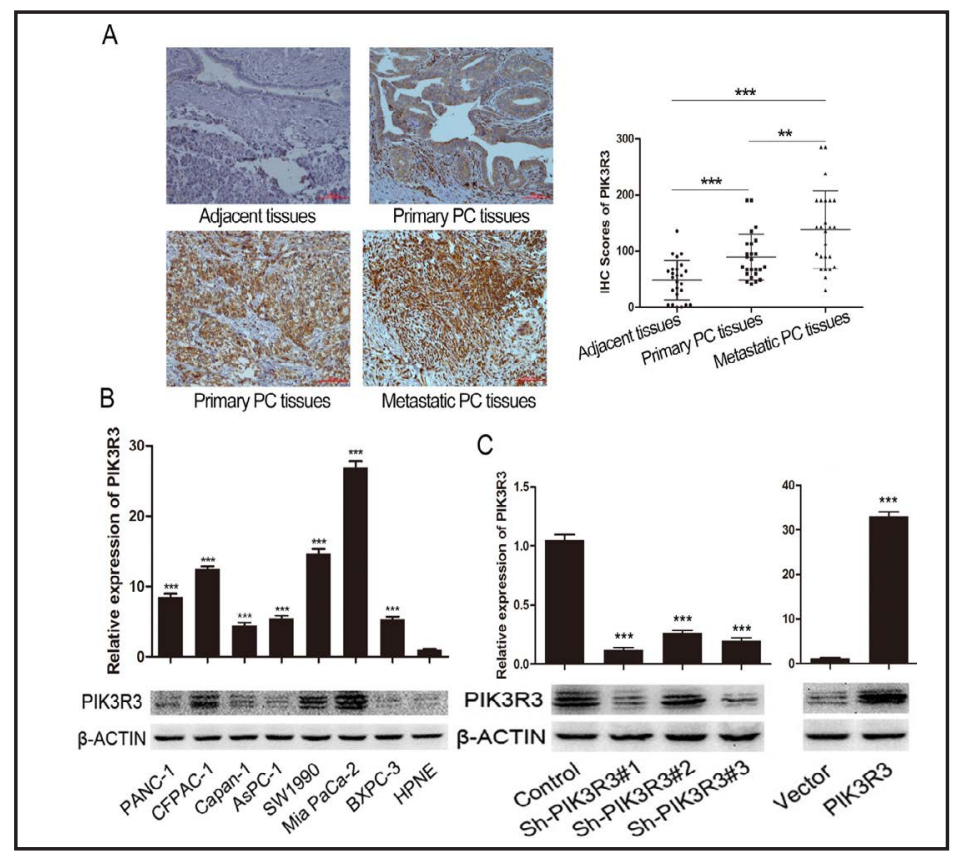

Fig. 1. The expression of PIK3R3 in PC. (A) Higher expression of PIK3R3 was observed in primary cancer tissues and metastatic tissues. (B) The expression of PIK3R3 was detected in PC cell lines. (C) The expression of PIK3R3 in stable knockdown and overexpression cell lines were confirmed by qRT-PCR (Upper) and Western blot (Lower). ** $\mathrm{P}<0.01$ and ${ }^{* * *} \mathrm{P}<0.001$.

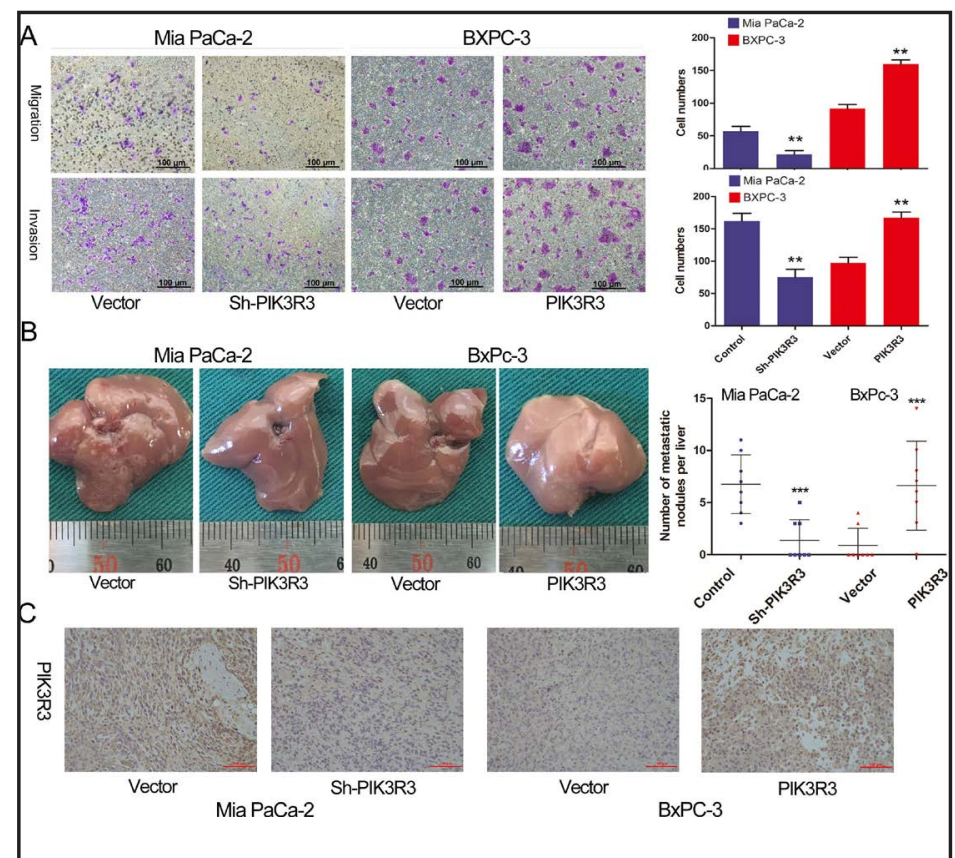

Fig. 2. PIK3R3 enhanced the migration and invasion of PC cells. (A) PIK3R3 knockdown and overexpression respectively inhibited and promoted the migration and invasion of PC cells in vitro. (B) The effects of PIK3R3 on PC cell migration and invasion was investigated in vivo. (C) The expression of PIK3R3 in PC cell xenografts was detected by IHC. ${ }^{* *} \mathrm{P}<0.01$. and ${ }^{* * *} \mathrm{P}<0.001$.

\section{KARGER}


migratory cells were significantly increased in PIK3R3 overexpression cells comparing to control cells, as well as invasive cells. However, the tendency of cell migration and invasion in PIK3R3 knockdown cells was contrary.

To further confirm the role of PIK3R3 in PC cell migration and invasion in vivo, we established an orthotopic pancreatic tumor model by implanting cancer cells in pancreas of the nude mice for 4 weeks. Metastatic nodules in liver were observed in 8 of $8 \mathrm{Mia} \mathrm{PaCa}-2$ Control cell implanted mice, 3 of 8 Mia PaCa-2-Sh-PIK3R3 cell implanted mice, 2 of 8 BxPc-3Vector cell implanted mice, and 7 of 8 BxPc-3-PIK3R3 cell implanted mice. Furthermore, the numbers of metastatic nodules per liver were significantly higher in PIK3R3 high expression cells (Mia PaCa-2-Control cells VS. Mia PaCa-2-Sh-PIK3R3 cell; BxPc-3-PIK3R3 cells VS. BxPc3-Vector cells) (Fig. 2B). The expression of PIK3R3 in tissues obtained from different mice models was also confirmed by IHC (Fig. 2C). Taken together, all results in this aspect revealed that PIK3R3 could promote the migration and invasion of PC cells in both vitro and vivo.

\section{PIK3R3 promotes EMT and ZEB1 expression of PC cells}

As mentioned in introduction, EMT was a metastatic mechanism for several cancers. Therefore, we formulated a hypothesis that whether PIK3R3 could promote PC cell migration and invasion via EMT.

We firstly assessed the EMT biomarkers in different PC cells by using Western blot and immunofluorescence, including E-Cadherin and Vimentin. As shown in Fig. 3A and $3 \mathrm{~B}$, Western blot and immunofluorescence data suggested that E-Cadherin protein level was decreased in BxPc-3-PIK3R3 cells in comparison with BxPc-3-Vector cells; however, E-Cadherin protein was not detected in Mia PaCa-2 related cells. Compared to control cells, Vimentin was down-regulated in Mia PaCa-2-Sh-PIK3R3 cells and up-regulated in BxPc-3PIK3R3. Furthermore, the tendency of E-Cadherin and Vimentin expression in xenografts were similar to that in cancer cells (Fig. 3C).

Although PIK3R3 could promote EMT in PC cells, the mechanisms involved in this process was absolutely unknown. EMT was commonly regulated by several transcription factors, such as SNAIL, SLUG, ZEB1, and ZEB2. Thus, these transcription factors were considered as mediators for the PIK3R3 induced EMT.

To test this hypothesis, we firstly evaluated the association between PIK3R3 expression and SNAIL, SLUG, ZEB1, and ZEB2 expression based on the data provided by TCGA (The Cancer Genome Atlas, http:// cancergenome.nih.gov/). The analytic data suggested that PIK3R3 expression was positively associated with SNAIL, ZEB1, and ZEB2 expression (Fig. 3D). However, only ZEB1 was up- or down-regulated in PIK3R3 overexpression or knockdown cells (Fig. 3E). These results indicated that PIK3R3 may induce EMT by increasing ZEB1 expression.

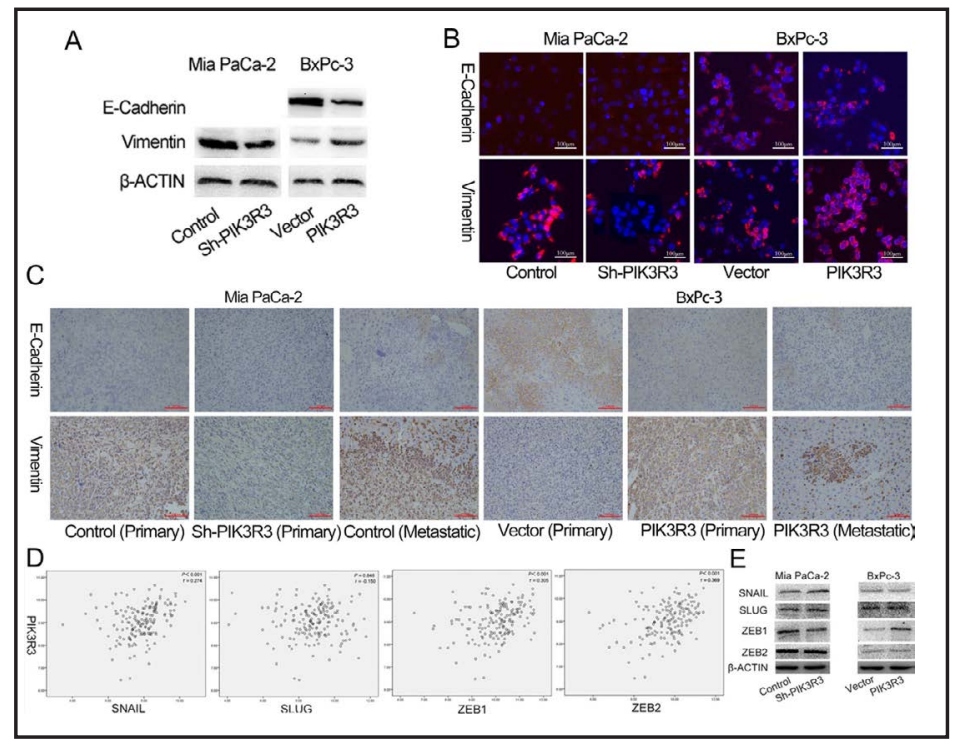

Fig. 3. PIK3R3 promoted the EMT process of PC cells. (A, B) Altered expression of E-Cadherin and Vimentin was detected in PIK3R3 knockdown and overexpression PC cells. (C) The results of IHC results confirmed the PIK3R3 mediated EMT biomarker expression. (D) PIK3R3 expression was positively associated with SNAIL, ZEB1, and ZEB2. (E) Only ZEB1 was regulated by PIK3R3. 
ERK1/2-ZEB1mediates PIK3R3 induced EMT

We knocked down ZEB1 expression in BxPc3-PIK3R3 cells to further investigate the role of ZEB1 by transiently transfecting ZEB1 SiRNA. The efficiency of ZEB1 SiRNA was shown in Fig. 4A. After silencing ZEB1 expression, the PIK3R3 overexpressioninduced EMT phenotype (E-Cadherin and Vimentin expression) of BxPc-3 cells was reversed (Fig. 4B and $4 \mathrm{C}$ ). Furthermore, the migration and invasion of BxPc-3-PIK3R3 cells were also decreased following the knockdown of ZEB1 (Fig. 4D). These results demonstrated that ZEB1 may mediate PIK3R3induced EMT phenotype and aggressive property.

Furthermore, ERK1/2ZEB1 pathway was widely reported to regulate the process of cancer cell EMT [25]. Therefore, we investigated the phosphorylation levels of ERK1/2 in PIK3R3 overexpression and knockdown cells. The ERK1/2 phosphorylation was respectively increased and decreased in overexpression and knockdown cells (Fig. 4E). Moreover, the inhibition of ERK1/2 by specific inhibitor (FR180204, 0.3mM) in BxPc-3-PIK3R3 cells resulted in the downregulation of ZEB1 expression, as well as the alternation of EMT phenotype (Fig. 4F).

\section{Discussion}

In this study, we found that PIK3R3 was overexpressed in PC tissues, especially in metastatic tissues. PIK3R3 overexpression could promote the migration and invasion of PC cells via ZEB1 upregulation induced EMT, while PIK3R3 knockdown could inhibit this phenomenon. Thus, PIK3R3 might be regarded as a potential therapeutic target to establish novel therapy strategies.

Previous studies suggested that the PIK3R3 is important in the regulation of several cell biological behaviors, such as proliferation, differentiation, cell cycle regulation, apoptosis, migration, and invasion [16, 26, 27]. More specifically, PIK3R3 could stimulate DNA synthesis and cell-cycle progression via its interaction with proliferation cell nuclear antigen (PCNA) [26]. PIK3R3 deficiency could lead to the decrease of proliferation of GC cells and induce 
G0/G1 cell cycle arrest by decreasing retinoblastoma protein (Rb) phosphorylation, and the expression of cyclin D1 and PCNA [15]. Blocking PIK3R3 signaling by Tat transactivator protein (TAT)-N24 could suppress the proliferation and induce the differentiation of leukemia cells [27]. Apoptosis induced by parquet was associated with decreased PIK3R3 expression and caspase activation [28]. Furthermore, PIK3R3 could promote cell migration and invasion in various types of cancer via different mechanisms [29], for example, this protein could enhance the migration and invasion of colorectal cancer by SLUG triggered EMT [19]. Similar to above-mentioned results, our data demonstrated that PIK3R3 could promote the migration and invasion of $\mathrm{PC}$ cells for the first time by experiments both in vitro and in vivo, and this function of PIK3R3 might be depending on the ZEB1 induced EMT.

EMT has been identified as a crucial step in the metastasis and invasion of malignancies $[30,31]$ EMT is characterized by the up-regulation of mesenchymal cell-specific marker proteins including $\mathrm{N}$-Cadherin and Vimentin, and the down-regulation of epithelial cellspecific marker proteins such as E-Cadherin [32]. In our study, our result suggested that mesenchymal cell-specific biomarker Vimentin expression were respectively increased and decreased in PIK3R3 overexpression and knockdown cells; however, epithelial cell-specific biomarkers expression E-Cadherin showed the opposite trend. These result indicated that PIK3R3 was a promotive factor for EMT of PC cells.

EMT is commonly activated by several embryonic and mesenchymal transcriptional factors. Some transcriptional factors could bind to the promoter region of E-Cadherin to suppress its expression, and further trigger the EMT conversion and metastasis of tumor cells, including SNAIL, SLUG, ZEB1 and ZEB2 [33, 34]. Among them, ZEB1 (characterized by the presence of 2 zinc finger clusters) is a well investigated transcriptional factor to act as a driver of EMT and cancer progression toward metastasis and invasion [35]. In our study, we have recognized ZEB1 as a downstream protein of PIK3R3 by evaluating the correlation between above-mentioned transcriptional factors and PIK3R3 expression based on the data obtained from TCGA, and assessing their expression in stable PIK3R3 overexpression and knockdown cells. Downregulation of ZEB1 in stable PIK3R3 overexpression cells could partially rescue EMT, migration, and invasion of pancreatic cancer cells. Obviously, the role of ZEB1 in cancer progression was further confirmed in this aspect. Furthermore, we also suggested that ERK1/2 participated in PIK3R3 mediated ZEB1 expression.

\section{Conclusion}

In conclusion, this is the first study to report that PIK3R3 was overexpressed in PC samples, and PIK3R3 could promote the migration and invasion of PC cells via enhancing ZEB1 mediated EMT. These results suggest that PIK3R3 may serve as an oncogene in PC pathogenesis and PIK3R3-targeted therapy for PC might be possible in future.

\section{Disclosure Statement}

The authors declare that they have no competing interests.

\section{Acknowledgements}

This work was supported by grants from the National Natural Science Foundation of China (81170336, 81272239, 81672449, 81672471), and the Research Innovation Program for College Graduates of Jiangsu Province (KYLX15_0954). Our research was also supported by the Innovation Capability Development Project of Jiangsu Province (BM2015004). 


\section{Cellular Physiology Cell Physiol Biochem 2018;46:1930-1938 \begin{tabular}{ll|l} 
DOI: 10.1159/000489382 & O 2018 The Author(s). Published by S. Karger AG, Basel \\
www.karger.com/cpb
\end{tabular}

\section{References}

1 Siegel RL, Miller KD, Jemal A: Cancer statistics, 2016 CA: Cancer J Clin 2016;66:7-30.

-2 Rahib L, Smith BD, Aizenberg R, Rosenzweig AB, Fleshman JM, Matrisian LM: Projecting cancer incidence and deaths to 2030: the unexpected burden of thyroid, liver, and pancreas cancers in the United States. Cancer Res 2014;74:2913-2921.

- 3 Strobel 0, Hank T, Hinz U, Bergmann F, Schneider L, Springfeld C, Jager D, Schirmacher P, Hackert T, Buchler MW: Pancreatic Cancer Surgery: The New R-status Counts. Ann Surg 2017;265:565-573.

4 Tuveson DA, Neoptolemos JP: Understanding metastasis in pancreatic cancer: a call for new clinical approaches. Cell 2012;148:21-23.

-5 Qian Y, Feng L, Wu W, Weng T, Hu C, Hong B, Wang FXC, Shen L, Wang Q, Jin X, Yao H. MicroRNA Expression Profiling of Pancreatic Cancer Cell Line L3.6p1 Following B7-H4 Knockdown. Cell Physiol Biochem 2017;44:494-504.

6 Yang W, Yang Y, Xia L, Yang Y, Wang F, Song M, Chen X, Liu J, Song Y, Zhao Y, Yang C. MiR-221 Promotes Capan-2 Pancreatic Ductal Adenocarcinoma Cells Proliferation by Targeting PTEN-Akt. Cell Physiol Biochem 2016;38:2366-2374.

7 Li D, Qu C, Ning Z, Wang H, Zang K, Zhuang L, Chen L, Wang P, Meng Z: Radiation promotes epithelialto-mesenchymal transition and invasion of pancreatic cancer cell by activating carcinoma-associated fibroblasts. Am J Cancer Res 2016;6:2192-2206.

8 Grunwald B, Vandooren J, Gerg M, Ahomaa K, Hunger A, Berchtold S, Akbareian S, Schaten S, Knolle P, Edwards DR, Opdenakker G, Kruger A: Systemic Ablation of MMP-9 Triggers Invasive Growth and Metastasis of Pancreatic Cancer via Deregulation of IL6 Expression in the Bone Marrow. Mol Cancer Res 2016;14:1147-1158.

-9 Heeg S, Das KK, Reichert M, Bakir B, Takano S, Caspers J, Aiello NM, Wu K, Neesse A, Maitra A, IacobuzioDonahue CA, Hicks P, Rustgi AK: ETS-Transcription Factor ETV1 Regulates Stromal Expansion and Metastasis in Pancreatic Cancer. Gastroenterology 2016;151:540-553 e514.

10 Cantelli G, Crosas-Molist E, Georgouli M, Sanz-Moreno V: TGFBeta-induced transcription in cancer. Semin Cancer Biol 2017;42:60-69.

11 Ohashi S, Natsuizaka M, Naganuma S, Kagawa S, Kimura S, Itoh H, Kalman RA, Nakagawa M, Darling DS, Basu D, Gimotty PA, Klein-Szanto AJ, Diehl JA, Herlyn M, Nakagawa H: A NOTCH3-mediated squamous cell differentiation program limits expansion of EMT-competent cells that express the ZEB transcription factors. Cancer Res 2011;71:6836-6847.

$12 \mathrm{Su}$ J, Wu S, Wu H, Li L, Guo T: CD44 is functionally crucial for driving lung cancer stem cells metastasis through Wnt/beta-catenin-FoxM1-Twist signaling. Mol Carcinog 2016;55:1962-1973.

13 Wang Z, Liu Z, Fang X, Yang H. MiR-142-5p Suppresses Tumorigenesis by Targeting PIK3CA in Non-Small Cell Lung Cancer. Cell Physiol Biochem 2017;43:2505-2515.

14 Yu T, Li J, Yan M, Liu L, Lin H, Zhao F, Sun L, Zhang Y, Cui Y, Zhang F, Li J, He X, Yao M: MicroRNA-193a-3p and -5p suppress the metastasis of human non-small-cell lung cancer by downregulating the ERBB4/PIK3R3/ mTOR/S6K2 signaling pathway. Oncogene 2015;34:413-423.

15 Zhou J, Chen GB, Tang YC, Sinha RA, Wu Y, Yap CS, Wang G, Hu J, Xia X, Tan P, Goh LK, Yen PM: Genetic and bioinformatic analyses of the expression and function of PI3K regulatory subunit PIK3R3 in an Asian patient gastric cancer library. BMC Med Genomics 2012;5:34.

-16 Cai JQ, Xu XW, Mou YP, Chen K, Pan Y, Wu D: Upregulation of HOXB7 promotes the tumorigenesis and progression of gastric cancer and correlates with clinical characteristics. Tumour Biol 2016;37:1641-1650.

17 Wang G, Chen C, Yang R, Cao X, Lai S, Luo X, Feng Y, Xia X, Gong J, Hu J: p55PIK-PI3K stimulates angiogenesis in colorectal cancer cell by activating NF-kappaB pathway. Angiogenesis 2013;16:561-573.

18 Xu L, Wen Z, Zhou Y, Liu Z, Li Q, Fei G, Luo J, Ren T: MicroRNA-7-regulated TLR9 signaling-enhanced growth and metastatic potential of human lung cancer cells by altering the phosphoinositide-3-kinase, regulatory subunit 3/Akt pathway. Mol Biol Cell 2013;24:42-55.

19 Wang G, Yang X, Li C, Cao X, Luo X, Hu J: PIK3R3 induces epithelial-to-mesenchymal transition and promotes metastasis in colorectal cancer. Mol Cancer Ther 2014;13:1837-1847.

20 Ouyang Y, Pan J, Qiang T, Ju J, Wang H: Transcriptomic changes associated with DKK4 overexpression in pancreatic cancer cells detected by RNA-Seq. Tumour Biol 2016;37:10827-10838. 


\section{Cellular Physiology Cell Physiol Biochem 2018;46:1930-1938 \begin{tabular}{l|l} 
DOI: 10.1159/000489382 & O 2018 The Author(s). Published by S. Karger AG, Basel \\
www.karger.com/cpb
\end{tabular}}

Peng et al.: PIK3R3 Promotes Metastasis of Pancreatic Cancer via ZEB1

21 Waddell N, Pajic M, Patch AM, Chang DK, Kassahn KS, Bailey P, Johns AL, Miller D, Nones K, Quek K: Whole genomes redefine the mutational landscape of pancreatic cancer. Nature 2015;518:495-501.

-22 Tang D, Yuan Z, Xue X, Lu Z, Zhang Y, Wang H, Chen M, An Y, Wei J, Zhu Y: High expression of Galectin-1 in pancreatic stellate cells plays a role in the development and maintenance of an immunosuppressive microenvironment in pancreatic cancer. Int J Cancer 2012;130:2337.

23 Peng YP, Xi CH, Zhu Y, Yin LD, Wei JS, Zhang JJ, Liu XC, Guo S, Fu Y, Miao Y: Altered expression of CD226 and CD96 on natural killer cells in patients with pancreatic cancer. Oncotarget 2016.

-24 Yu Y, Xiao CH, Tan LD, Wang QS, Li XQ, Feng YM: Cancer-associated fibroblasts induce epithelialmesenchymal transition of breast cancer cells through paracrine TGF-beta signalling. Br J Cancer 2014;110:724-732.

25 Ichikawa K, Kubota Y, Nakamura T, Weng JS, Tomida T, Saito H, Takekawa M: MCRIP1, an ERK substrate, mediates ERK-induced gene silencing during epithelial-mesenchymal transition by regulating the corepressor CtBP. Mol Cell 2015;58:35-46.

26 Wang G, Cao X, Lai S, Luo X, Feng Y, Xia X, Yen PM, Gong J, Hu J: PI3K stimulates DNA synthesis and cell-cycle progression via its p55PIK regulatory subunit interaction with PCNA. Mol Cancer Ther 2013;12:21002109.

27 Wang G, Deng Y, Cao X, Lai S, Tong Y, Luo X, Feng Y, Xia X, Gong J, Hu J: Blocking p55PIK signaling inhibits proliferation and induces differentiation of leukemia cells. Cell Death Differ 2012;19:1870-1879.

28 Zhou Y, Li F, Tian X, Wang B, Ding M, Pang H: Changes in phosphatidylinositol 3-kinase 55 kDa gamma expression and subcellular localization may be caspase 6 dependent in paraquat-induced SH-SY5Y apoptosis. Hum Exp Toxicol 2014;33:761-771.

29 Cao G, Dong W, Meng X, Liu H, Liao H, Liu S: MiR-511 inhibits growth and metastasis of human hepatocellular carcinoma cells by targeting PIK3R3. Tumour Biol 2015;36:4453-4459.

-30 Toth DP, Guenther LC, Shum DT: Proliferating cell nuclear antigen (PCNA); prognostic value in the clinical recurrence of primary basal cell carcinoma. J Dermatol Sci 1996;11:36-40.

-31 Acharya N, Yoon JH, Gali H, Unk I, Haracska L, Johnson RE, Hurwitz J, Prakash L, Prakash S: Roles of PCNAbinding and ubiquitin-binding domains in human DNA polymerase eta in translesion DNA synthesis. Proc Natl Acad Sci U S A 2008;105:17724-17729.

32 Wang QS, Kong PZ, Li XQ Yang F, Feng YM: FOXF2 deficiency promotes epithelial-mesenchymal transition and metastasis of basal-like breast cancer. Breast Cancer Res 2015;17:30.

-33 Fang C, Zan J, Yue B, Liu C, He C, Yan D: Long Noncoding RNA ZFAS1 promotes the progression of colonic cancer by modulating ZEB1 expression. J Gastroenterol Hepatol 2017;32:1204-1211.

34 Amoroso MR, Matassa DS, Agliarulo I, Avolio R, Lu H, Sisinni L, Lettini G, Gabra H, Landriscina M, Esposito F: TRAP1 downregulation in human ovarian cancer enhances invasion and epithelial-mesenchymal transition. Cell Death Dis 2016; 7:e2522.

35 Zhang L, Zhang W, Li Y, Alvarez A, Li Z, Wang Y, Song L, Lv D, Nakano I, Hu B, Cheng SY, Feng H: SHP-2upregulated ZEB1 is important for PDGFRalpha-driven glioma epithelial-mesenchymal transition and invasion in mice and humans. Oncogene 2016;35:5641-5652. 